\title{
A TREM2 dependent control of Microglial and Astrocytic responses in a mouse model of Alzheimer's Disease
}

\author{
Nnamdi Achebe ${ }^{1}$, Shweta S. Puntambekar (Ph.D) ${ }^{2}$, Bruce T. Lamb (Ph.D) ${ }^{2}$ \\ ${ }^{1}$ Indiana University School of Medicine, ${ }^{2}$ Indiana University School of Medicine, \\ Department of Medical and Molecular Genetics, Stark Neurosciences Research \\ Institute.
}

\begin{abstract}
Background and Hypothesis:
Alzheimer's Disease (AD) is characterized by deposition of extracellular amyloid beta plaques resulting in activation of plaque associated CNS glial cells, namely microglia and astrocytes. While extensive research has been directed to understand the role of microglia in neurodegenerative changes in $A D$, relatively little is known of astrocytic contributions to the disease. Studies using nerve injury models have shown that pro-inflammatory microglia can induce neurotoxic astrocytic phenotypes. In AD, the microglial gene TREM2 has been implicated in mediating neuroprotective immune functions. Research suggests that microglia go through TREM2-independent, followed by TREM2-dependent activation to mediate neuroprotection in diseases like AD, ALS and PD. The central objective of this project is to evaluate whether TREM2 dependent skewing of microglial activation generates neuro-protective/toxic astroglial populations.
\end{abstract}

\section{Experimental Design or Project Methods:}

We will use the 5XFAD mouse model of $A D$ in this project. Changes in microglial and astroglial activation will be evaluated in 4-month-old 5xFAD; TREM2 ${ }^{+/}$mice using immunofluorescence and confocal microscopy

\section{Results:}

Our results show an increase in proinflammatory, plaque associated microglia expressing $\mathrm{MHCll}$ and $\mathrm{C} 1 \mathrm{qA}$ in 5XFAD;TREM2+/- mice relative to 5XFAD;TREM2+/+ mice. This is accompanied by an increase in GFAP+S100Breactive astrocytes in the hippocampus and subiculum.

\section{Conclusion and Potential Impact:}

TREM2 deficiency in a mouse model of AD skews the microglia to a proinflammatory phenotype, which may correlate with heightened astroglial reactivity and neurotoxicity. As loss of function mutations in TREM2 have been associated with an increased risk of neurodegenerative changes, these data shed light on how TREM2-mediated microglial-astrocytic crosstalk may influence disease progression. 